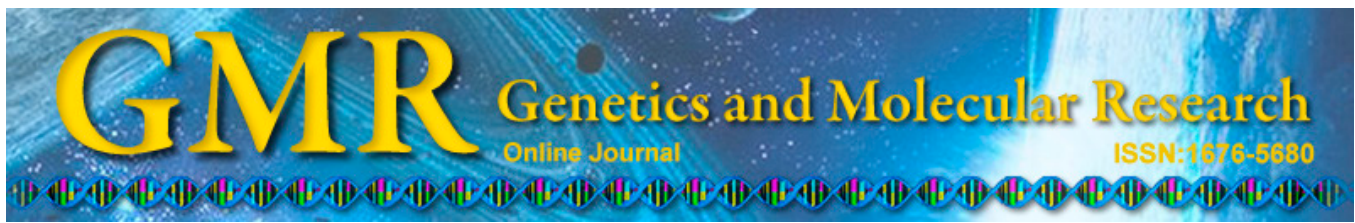

Case Report

\title{
Serious pulmonary infection in a splenectomized patient with adult type 1 Gaucher disease
}

\author{
Y. Zhang*, Y.F. Mao* and J.M. Du \\ Department of Anesthesiology and Surgical Intensive Care Unit, \\ Xinhua Hospital, Shanghai Jiao Tong University School of Medicine, \\ Shanghai, China \\ *These authors contributed equally to this study. \\ Corresponding author: J.M. Du \\ E-mail: dujmin@126.com
}

Genet. Mol. Res. 14 (2): 3338-3344 (2015)

Received June 27, 2014

Accepted October 28, 2014

Published April 13, 2015

DOI http://dx.doi.org/10.4238/2015.April.13.13

\begin{abstract}
A 49-year-old man with a history of Gaucher disease type 1, resulting in serious splenomegaly and eating disorder, was referred to our department and underwent a splenectomy under general anesthesia. Gaucher disease is very rare, and its first signs are unexplained splenomegaly and hypersplenism. On preoperative examination, the patient's platelet count was slightly low, and his other test results were normal. Surprisingly, on the first postoperative day, the patient developed a lung infection. This gradually progressed to acute respiratory distress syndrome with respiratory failure, requiring intubation and mechanical ventilation. The patient eventually recovered, and he was discharged after receiving antibiotics and other treatments to enhance immunity. However, his postoperative lung infection led to a significantly prolonged and expensive hospital stay. This case suggests that we must pay close attention to the immune dysfunction of patients with Gaucher disease type 1. Anesthesia and surgery with accompanying post-traumatic stress can weaken patients' immunity
\end{abstract}


and cause susceptibility to severe lung infections. Pulmonary signs and functions should be monitored closely during the perioperative period, and, if necessary, gamma globulin and thymosin should be administered early in the preoperative or postoperative period to enhance immunity.

Key words: Gaucher disease type 1; Serious pulmonary infection; Splenectomy

\section{INTRODUCTION}

Gaucher's disease, also known as glucocerebrosidase deficiency, is an autosomal recessive lysosomal storage disease. It was first named by Gaucher in 1882 (Jmoudiak and Futerman, 2005) and is clinically very rare. Type 1 is its most common form, and patients with type 1 may require splenectomies because of serious splenomegaly and hypersplenism. Although some research suggests that the proliferation of Gaucher cells may cause lung tissue to have a higher susceptibility to infection, no clinical studies have reported on the monitoring and protection of lung function during the perioperative period. An adult male patient with Gaucher disease type 1 presented to our department for a splenectomy and developed a postoperative pulmonary infection that rapidly progressed to acute respiratory distress syndrome. The case report of his treatment follows.

\section{MATERIAL AND METHODS}

\section{Case report}

A 49-year-old man with congenital Gaucher disease type 1 had been treated for a long time with imiglucerase. Because he developed severe symptomatic splenomegaly, he was referred for a splenectomy.

Preoperative examination revealed the following results: 1) on ultrasonography, severe swelling of the spleen; 2) on radiography, elevated right diaphragm with no obvious lung inflammation (Figure 1A); 3) on routine blood tests, complete blood count showed white blood cell count (WBC), $8.1 \times 10^{9} / \mathrm{L}$; neutrophil percentage (N\%), 90.6\%; hemoglobin ( $\left.\mathrm{Hb}\right)$, $70 \mathrm{~g} / \mathrm{L}$; and platelet count (PLT), $237 \times 10^{9} / \mathrm{L}$.

Liver, kidney, and coagulation function were all normal. On the second day after admission, the patient underwent a splenectomy under general anesthesia. Postoperatively, the patient was administered routine treatment, including cefuroxime sodium for infection prophylaxis and pantoprazole for prevention of stress ulcer.

On the second postoperative day, the patient appeared dyspneic, with deep respirations at a rate of approximately $30 \mathrm{breaths} / \mathrm{min}$. He was administered oxygen via nasal cannula at $6 \mathrm{~L} / \mathrm{min}$, and oxygen saturation $\left(\mathrm{SaO}_{2}\right)$ was measured at $90-95 \%$. The patient's temperature was $37.4^{\circ} \mathrm{C}$. Rough breath sounds could be heard in both lungs, and coarse rales were audible in the right upper lobe. An arterial blood gas (ABG) revealed $\mathrm{pH}, 7.45 ; \mathrm{PCO}_{2}, 4.42 \mathrm{kPa}$; and $\mathrm{PO}_{2}, 10.6 \mathrm{kPa}$. Chest radiography showed lung inflammation and right lung consolidation (Figure 1B). Procalcitonin (PCT) level was $4.47 \mathrm{ng} / \mathrm{mL}$, and complete blood count showed WBC, $15.1 \times 10^{9} / \mathrm{L} ; \mathrm{N} \%, 90.6 \%$;b, $70 \mathrm{~g} / \mathrm{L}$; and PLT, $237 \times 10^{9} / \mathrm{L}$. While awaiting sputum culture results, we started noninvasive mechanical ventilation and empirical antibiotic treat- 
ment. Specifically, the patient was administered intravenous meropenem $(1.0 \mathrm{~g} \mathrm{q} 12 \mathrm{~h})$ for Gram-negative bacteria and intravenous teicoplanin ( $0.2 \mathrm{~g}$ daily) for Gram-positive bacteria.

After 6 days, the patient's pulmonary symptoms worsened. $\mathrm{His}_{\mathrm{SaO}}$ was $87-95 \%$ under noninvasive mechanical ventilation with fraction of inspired oxygen $80 \%$, and his temperature was $37.9^{\circ} \mathrm{C}$. Coarse rales could be heard in both lungs, with decreased breath sounds in the right lung. An ABG showed $\mathrm{pH}, 7.49 ; \mathrm{PCO}_{2}, 3.76 \mathrm{kPa}$; and $\mathrm{PO}_{2}, 7.6 \mathrm{kPa}$. Both chest radiograph and computed tomography scan showed patchy shadows of progressively increasing density and atelectasis in the lower lobes because of hydrothorax (Figure 1C, Figure 2A).

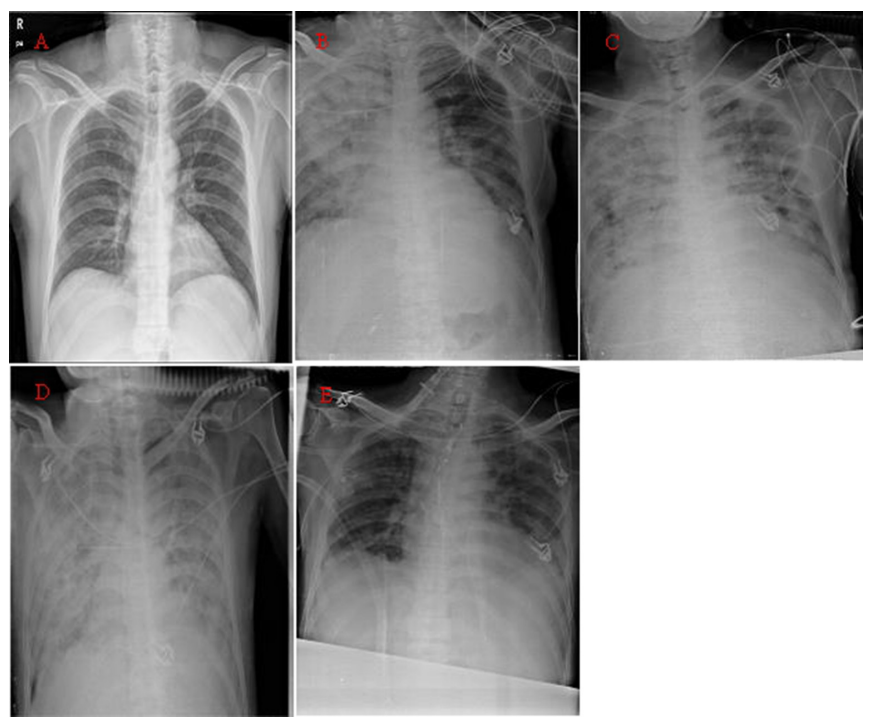

Figure 1. Changes on the patient's chest radiograph. A. Radiograph before surgery shows elevation of the right diaphragm with no obvious lung inflammation. B. Radiograph on the second postoperative day shows lung inflammation and right lung consolidation. C. Radiograph on the sixth postoperative day shows patchy shadows of progressively increasing density. D. Radiograph on the 10th postoperative day shows that the range of patchy shadows is widening. E. Radiograph on the 33rd postoperative day shows that the infection has resolved.

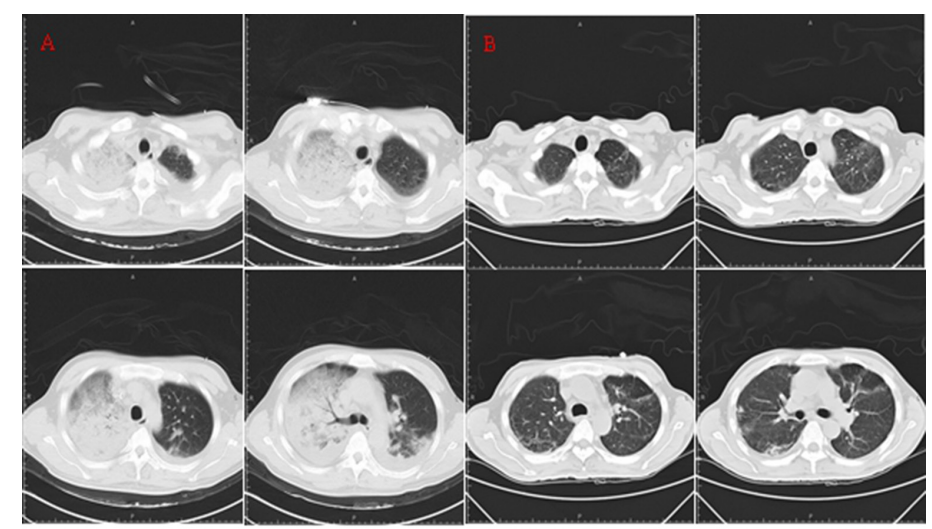

Figure 2. Changes on the patient's chest computed tomography (CT) scan. A. Serious right lung inflammation and atelectasis are visible on the chest CT scan on the sixth postoperative day. B. Chest CT scan after extubation. 
The PCT level was $0.78 \mathrm{ng} / \mathrm{mL}$, and complete blood count showed WBC, $12 \times 10^{9} / \mathrm{L}$; $\mathrm{N} \%, 88.3 \%$; Hb, $90 \mathrm{~g} / \mathrm{L}$; hematocrit, 0.26 ; and PLT, $415 \times 10^{9} / \mathrm{L}$. We started antifungal treatment with intravenous caspofungin (50 $\mathrm{mg}$ daily), antiviral treatment with intravenous ganciclovir (250 mg q12 h), and treatment for atypical pathogens with oral sulfamethoxazole compound tablets (SMZ). We also administered intravenous human immunoglobulin (10 g daily) and thymosin to enhance the patient's immunity.

On the tenth postoperative day, the patient's symptoms continued to worsen to the point that we could not maintain his oxygenation, and a radiograph showed that the range of patchy shadows in the lungs was widening (Figure 1D). Endotracheal intubation was performed, and the patient was continued on mechanical ventilation. His sputum culture came back positive for Acinetobacter baumannii, and on the basis of the drug sensitivities, we adjusted his antibiotic treatment to include intravenous meropenem $(1.0 \mathrm{~g} \mathrm{q} 12 \mathrm{~h})$, intravenous amikacin ( $0.8 \mathrm{~g}$ daily), oral SMZ, and intravenous amoxicillin/sulbactam (3.0 g q8 h).

After the change in antibiotics, the patient's condition gradually stabilized, and his oxygenation gradually improved. The patient was successfully extubated 33 days after his surgery (Figures $1 \mathrm{E}$ and $2 \mathrm{~B}$ ). He ultimately recovered, and he was discharged on the 58th postoperative day.

\section{DISCUSSION}

The cause of Gaucher disease is deficiency of $\beta$-glucocerebrosidase, which leads to the accumulation of glucocerebroside released from the aging erythrocyte, and leukocytes and platelets accumulate in lysosomes of mononuclear macrophages through the reticuloendothelial system in different organs. This occurs because glucocerebroside cannot be hydrolyzed to glucose and N-acyl-sphingosine, creating the Gaucher cell (Liel et al., 1994). The accumulation of Gaucher cells in various tissues leads to damage of multiple organ systems. Accumulation in liver and spleen causes hepatosplenomegaly and hypersplenism; in lungs, it can lead to repeated pulmonary infections; in bone marrow, it can affect hematopoietic function and cause bone destruction (osteoporosis, frequently); in the nervous system, it can result in growth retardation and corresponding nerve dysfunction (Pastores, 2010; Rosenbloom and Weinreb, 2013). Clinically, Gaucher disease is divided into 3 types according to central nervous system (CNS) symptoms: type 1 (adult) is most common, is non-neuropathic, and has a good prognosis; type 2 (acute infant mental derangement variant) is acute and severe with onset within 1 year of age and has serious CNS symptoms and poor prognosis, with death occurring within 2 years; type 3 (subacute neuropathy) is characterized primarily by epileptic seizures that start at around age 10 years and has a poor prognosis, with death often occurring in childhood or adolescence (Stirnemann et al., 2003).

Our patient's Gaucher disease was type 1, in which the onset is subtle and slow, and clinical symptoms are not obvious. The first sign is unexplained splenomegaly and hypersplenism, which can cause mechanical compression of lung tissue and lead to lung disease (Schneider et al., 1977; Kerem et al., 1996). Although pulmonary symptoms are not so obvious and lung function is not significantly affected, pathological changes in the lungs are visible because of the deposition of Gaucher cells. According to an autopsy report by Lee and Yousem (1988), 30\% of patients with Gaucher disease have obvious pathological lung changes, such as: 1) Gaucher cell deposition causing fibrosis around bronchi, capillaries, and alveoli; 2) pulmonary hypertension due to Gaucher cell deposition and pressure on pulmonary capillaries; 
and 3) chronic inflammatory invasion of pulmonary alveoli. Because the disease progression is variable, corresponding signs and symptoms vary and may include pulmonary hypertension, pulmonary interstitial lesions, and hypoxemia (mostly due to increased lung shunting) (Lee and Yousem, 1988). On the basis of clinical manifestations and abnormal bone marrow morphology (presence of Gaucher cells), a preliminary diagnosis of Gaucher disease can be made, but a definitive diagnosis should be based on $\beta$-glucocerebrosidase activity or DNA detection (Pastores and Hughes, 2000; Mankin et al., 2001).

The exact pathophysiological mechanism of Gaucher disease is not very clear. Recent research suggests that excessive glucocerebroside accumulation is the main factor that leads to activation of mononuclear macrophages and the inflammatory reaction (Burstein et al., 1987; Aker et al., 1993). However, this theory is flawed. Maródi et al. (1995) found that the bactericidal activity of mononuclear macrophages and superoxide anion $\left(\mathrm{O}_{2}^{-}\right)$release in Gaucher disease patients was much lower than in a control group. These studies indicate that the immune system function of patients with Gaucher disease is defective. Undoubtedly, Gaucher cell deposits in lung tissue cause pathological changes and deficient immune cell function so that patients with Gaucher disease are susceptible to pulmonary infections (Machaczka et al., 2014). Through analysis of this case of a middle-aged male patient, we concluded that the main reason for the lung infection shortly after splenectomy might have been the traumatic stress of surgery and anesthesia, which caused the patient's immunity to further decline. The patient's postoperative treatment was mainly directed at fighting infection and providing immune support.

Regarding anti-infection treatment, the patient had no obvious signs of infection on admission, and when the lung infection and hypoxemia developed on the second postoperative day, the diagnosis was hospital-acquired pneumonia. According to the guidelines of the American Thoracic Society (American Thoracic Society, 2005), carbapenems are preferred for treatment of hospital-acquired pneumonia; therefore, we chose to initiate treatment with meropenem. A chest radiograph showed bilateral lung involvement, and compared with the radiograph of the day before, lesions had expanded more than 50\% within 24h. The patient's oxygen index was 167 . These findings were consistent with the clinical manifestations of severe pneumonia. Therefore, we started teicoplanin empirically as combination treatment for Gram-positive bacteria. However, the patient's condition continued to worsen. We then added SMZ combined with antifungal and antiviral drugs, and the patient was administered human immunoglobulin and thymosin to enhance his immunity. The lung infection continued to expand based on the radiography reports, which showed progression from the right upper lung to the whole right lung and then to the left lung after 10 days of treatment. At that point, the results of the sputum culture came back positive for A. baumannii. On the basis of the drug sensitivities, we changed the antibiotic treatment to a combination of meropenem, amikacin, SMZ, and amoxicillin/sulbactam. After several days, the patient's temperature gradually returned to normal, leukocyte and neutrophil counts returned to normal, and the chest radiography findings improved. We considered that the pulmonary infection might have been caused by $A$. baumannii, which is a conditional pathogenic bacterium. However, in $50 \%$ of patients who develop infections after splenectomy, the pathogenic bacterium is pneumococcus (Holdsworth et al., 1991). Therefore, the possibility of a mixed postoperative infection cannot be excluded.

Regarding immune support treatment, human immunoglobulin (PH4) by intravenous injection is a kind of passive immunotherapy (Chinen et al., 2014). It can increase the levels of immunoglobulins, which can enhance the patient's antibody response, neutralizing toxins directly and killing both bacteria and viruses, so that it enhances patient's immunity (Ale- 
jandria et al., 2013; Chinen et al., 2014). The purpose of giving thymosin is to stimulate and adjust a patient's active immunity (Zhang et al., 2005). Its actions may include the following: 1) stimulate lymphocyte mitogen from peripheral blood to promote maturity of T cells; 2) increase interferon- $\alpha$ and interferon- $\gamma$ secreted by T cells; 3 ) increase cytokines, such as interleukin; and 4) strengthen natural killer cell activity (Kaminsky et al., 1985; Chandratilleke and Marsh, 2000; Merlino and Marsh, 2001; Zhang et al., 2005). Because immunity declines after splenectomy, increasing patients' susceptibility to severe infections, it is important to provide immune support treatment. However, because of our patient's economic situation, we did not administer preoperative and early postoperative immune support treatment and only resorted to this when the infection became severe and antibiotics were ineffective. Multi-resistant $A$. baumannii is a conditioned pathogen; thus, it may effectively respond to immune support treatment.

On the basis of this case, we concluded that administration of immune support treatment, such as intravenous human immunoglobulin, to patients before they undergo splenectomy may be effective for preventing infections, and the concept of asepsis must be strengthened to reduce the occurrence of opportunistic infections. Dynamic monitoring of pulmonary function, such as with ABGs, chest radiography, and sputum cultures, will lead to timely detection and, therefore, timely treatment of any infections. In the event of a lung infection, wide-spectrum antibiotics should be administered empirically, with subsequent adjustment of antibiotics based on the results of sputum cultures. While awaiting culture results, patients should be administered human immunoglobulin and thymosin to enhance their passive or active immunity. Additionally, attention must be given to basic monitoring and treatment measures, such as hepatic/renal function, coagulation function, water and electrolyte balance, and nutritional support. All these are vital for maintaining patients' vital signs and other physiological parameters in the normal or near-normal range.

\section{Conflicts of interest}

The authors declare no conflict of interest.

\section{ACKNOWLEDGMENTS}

Research supported by the Researchers Fund of Xinhua Hospital Affiliated with Shanghai Jiao Tong University School of Medicine (\#13YJ21).

\section{REFERENCES}

Aker M, Zimran A, Abrahamov A, Horowitz M, et al. (1993). Abnormal neutrophil chemotaxis in Gaucher disease. $B r$. J. Haematol. 83: 187-191.

Alejandria MM, Lansang MA, Dans LF and Mantaring JB 3rd (2013). Intravenous immunoglobulin for treating sepsis, severe sepsis and septic shock. Cochrane Database Syst. Rev. 9: CD001090.

American Thoracic Society; Infectious Diseases Society of America (2005). Guidelines for the management of adults with hospital-acquired, ventilator-associated, and healthcare-associated pneumonia. Am. J. Respir. Crit. Care Med. 171: 388-416.

Burstein Y, Zakuth V, Rechavi G and Spirer Z (1987). Abnormalities of cellular immunity and natural killer cells in Gaucher's disease. J. Clin. Lab. Immunol. 23: 149-151.

Chandratilleke D and Marsh JA (2000). The effect of thymulin on avian IL-2 receptor expression. Int. J. Immunopharmacol. 22: 887-896.

Chinen J, Notarangelo LD and Shearer WT (2014). Advances in basic and clinical immunology in 2013. J. Allergy Clin. 
Immunol. 133: 967-976.

Holdsworth RJ, Irving AD and Cuschieri A (1991). Postsplenectomy sepsis and its mortality rate: actual versus perceived risks. Br. J. Surg. 78: 1031-1038.

Jmoudiak M and Futerman AH (2005). Gaucher disease: pathological mechanisms and modern management. $B r . J$. Haematol. 129: 178-188.

Kaminsky SG, Nakamura I and Cudkowicz G (1985). Genetic control of the natural killer cell activity in SJL and other strains of mice. J. Immunol. 135: 665-671.

Kerem E, Elstein D, Abrahamov A, Bar Ziv Y, et al. (1996). Pulmonary function abnormalities in type I Gaucher disease. Eur. Respir. J. 9: 340-345.

Lee RE and Yousem SA (1988). The frequency and type of lung involvement in patients with Gaucher's disease. Lab. Invest. 58: A54.

Liel Y, Rudich A, Nagauker-Shriker O, Yermiyahu T, et al. (1994). Monocyte dysfunction in patients with Gaucher disease: evidence for interference of glucocerebroside with superoxide generation. Blood 83: 2646-2653.

Machaczka M, Lorenz F, Kleinotiene G, Bulanda A, et al. (2014). Recurrent pulmonary aspergillosis and mycobacterial infection in an unsplenectomized patient with type 1 Gaucher disease. Ups. J. Med. Sci. 119: 44-49.

Mankin HJ, Rosenthal DI and Xavier R (2001). Gaucher disease. New approaches to an ancient disease. J. Bone Joint Surg. Am. 83-A: 748-762.

Maródi L, Káposzta R, Tóth J and László A (1995). Impaired microbicidal capacity of mononuclear phagocytes from patients with type I Gaucher disease: partial correction by enzyme replacement therapy. Blood 86: 4645-4649.

Merlino PG and Marsh JA (2001). Evidence for the direct action of thymulin on avian NK cells. Dev. Comp. Immunol. 25: 337-343.

Pastores GM (2010). Neuropathic Gaucher disease. Wien. Med. Wochenschr. 160: 605-608.

Pastores GM and Hughes DA (2000). Gaucher Disease. In: GeneReviews ${ }^{\circledR}$ [Internet] (Pagon RA, Adam MP, Bird TD, Dolan CR, Fong CT, Smith RJH and Stephens K, eds.). University of Washington, Seattle; 1993-2014 [updated 2013 Sep 19].

Rosenbloom BE and Weinreb NJ (2013). Gaucher disease: a comprehensive review. Crit. Rev. Oncog. 18: 163-175.

Schneider EL, Epstein CJ, Kaback MJ and Brandes D (1977). Severe pulmonary involvement in adult Gaucher's disease. Report of three cases and review of the literature. Am. J. Med. 63: 475-480.

Stirnemann J, Caubel I, Kettaneh A, Fain O, et al. (2003). Epidemiologic, clinical, biological and therapeutic aspects of Gaucher disease. Presse Med. 32: 503-511.

Zhang P, Chan J, Dragoi AM, Gong X, et al. (2005). Activation of IKK by thymosin alpha1 requires the TRAF6 signalling pathway. EMBO Rep. 6: 531-537. 